\title{
The effects of sweeteners and sweetness enhancers on obesity and diabetes: a review
}

\begin{abstract}
Yanli Jiao and Yu Wang*
Citrus Research and Education Center, Food Science and Human Nutrition, University of Florida, 700 Experiment Station Road, Lake Alfred, FL 33850, USA

*Corresponding author: Yu Wang, Citrus Research and Education Center, Food Science and Human Nutrition, University of Florida, 700 Experiment Station Road, Lake Alfred, FL 33850, USA. E-mail: yu.wang@ufl.edu

DOI: $10.31665 / J F B .2018 .4166$

Received: December 18, 2018; Revised received \& accepted: December 23, 2018

Citation: Jiao, Y., and Wang, Y. (2018). The effects of sweeteners and sweetness enhancers on obesity and diabetes: a review. J. Food Bioact. 4: 107-116.

Abstract

Sweet taste, one of the five basic taste qualities, is not only important for evaluation of food quality, but also guides the dietary food choices of animals. Sweet taste involves a variety of chemical compounds and structures, including natural sugars, sugar alcohols, natural and artificial sweeteners, and sweet-tasting proteins. The preference for sweetness has induced the over-consumption of sugar, contributing to certain prevailing health problems, such as obesity, diabetes and cardiovascular disease. Non-nutritive sweeteners, including natural and synthetic sweeteners, and sweet-tasting proteins have been added to foods to reduce the caloric intake from sugar, but many of these sugar substitutes induce an off-taste or after taste that negatively impacts any pleasure derived from the sweet taste. Sweet taste is detected by sweet taste receptor, that also play an important role in the metabolic regulation of the body, such as glucose homeostasis and incretin hormone secretion. In this review, the role of sweet tastants and the sweet taste receptors involved in sweetness perception, and their effect on obesity and diabetes are summarized. Sweet taste enhancement, as a new way to solve the over-consumption of sugar, is discussed in this contribution. Sweet taste enhancers can bind with sweet tastans to potentiate the sweetness of food without producing any taste by itself. Various type of sweet taste enhancers, including synthetic compounds, food-processed substances and aroma compounds, are summarized. Notably, few natural, non-volatile compounds have been identified as sweetness enhancers.
\end{abstract}

Keywords: Sweetness; Sweet taste receptors; Sweet taste enhancer; Diabetes; Obesity.

\section{Introduction}

The sense of taste is very important for animals and humans to evaluate both food quality and to select proper nutrients for survival (Horio et al., 2010). Animals prefer a sweet taste, because the sense of sweetness implies a potential for caloric intake derived from a source full of nutrients (Jiang et al., 2005). Additionally, sweetness is a palatable and pleasurable sense that guides the dietary food choices of animals. Sweet taste involves a variety of chemical compounds with varied structures, including natural sug- ars, sugar alcohols, natural and artificial sweeteners, and sweettasting proteins (Belloir et al., 2017). Consequently, habitual sugar consumption is linked to the prevalence of numerous public health problems. For decades, epidemiological studies have demonstrated that sugar over-consumption poses a serious threat to human health, e.g. overweight, obesity, diabetes and cardiovascular diseases. Sugar-sweetened food and beverages are the major dietary sources of sweet taste in human, especially Americans (Huang et al., 2014). In the $19^{\text {th }}$ century, average annual sugar consumption was $2.5 \mathrm{~kg}$ per person in the US. This number increased to $68 \mathrm{~kg}$ by the beginning of $21^{\text {st }}$ century ( $\mathrm{Li}$ et al., 2011). Even with a de- 
creased trend of sugar consumption in recent decades, this number is still much higher than the recommended dietary upper limit in US diet, especially among children (Yang et al., 2014). It has been recommended to decrease added-sugar intake to less than 10 percent of total daily calories in order to reduce public health problems in the US (U.S. Department of Health and Human, 2015). Controlling sugar intake and reducing calories from sugar intake are quite difficult.

The food industries have already substituted numerous alternatives, such as sugar alcohol, non- or low-calorie sweeteners, to reduce the caloric intake from sugar (Beltrami et al., 2018). To perceive sweetness, the sweet taste receptor interacts with imbibed sweet tastants. Sweeteners provide a high sweet taste sensation while reducing the calories in the final product. However, most of these substitutes fail to induce a 'real' sugar taste. Commonly they result in an off-taste or aftertaste, such as bitter, metallic or licorice-like, or slow or delay the sweetness onset (DuBois and Prakash, 2012; Li et al., 2011). Moreover, some may potentially cause adverse effects, including weight gain, central adiposity, mental disorders, bladder cancer and psychological problems (Sylvetsky et al., 2016). To address these problems, a new strategy of positive allosteric modulators (PAMs) has been proposed. PAMs, including sweetness enhancer, can bind at the allosteric sites of sweet taste receptors and enhance receptor activity (DuBois and Prakash, 2012). Sweetness enhancers do not taste sweet by themselves, but can preserve and potentiate the desirable sweetness intensity of sweet tastants in such a way that the amount of sweet tastant used in the diet is reduced, thus decreasing caloric intake, as well as modifying or inhibiting any undesirable taste (Servant et al., 2010). Additionally, sweet taste receptors are involved in glucose homeostasis and incretin hormone secretion, consequently playing an important role in physiological regulation and providing a therapeutic method to treat obesity and diabetes (Neiers et al., 2016).

\section{Sweeteners, obesity and diabetes}

In recent decades, there has been an increase in the overweight and obese population. In the US, body mass index (BMI), an important index to evaluate tissue mass, has recently increased to $35 \%$ for men and $40 \%$ for women (Chia et al., 2016). Approximately two thirds of American adults are considered overweight, whereas one third are classified as obese (Smith et al., 2016). Sugar, a common sweet tastant in the daily diet, is a major factor contributing to various health problems, including obesity, metabolic syndrome, diabetes and cardiovascular disease (Shankar et al., 2013). The correlation between sugar-added food and beverage and arising health problems have been the subject of many studies. Some epidemiological studies have demonstrated that consumption of sugarsweetened food may be a driving factor behind long-term fat and weight gain, due to the high energy intake from sugar (Fowler et al., 2008). Significant consumption of large amounts of sugar, such as sucrose, independently increases the risk of becoming overweight and obese (Stanhope, 2016). Additionally, sugar sweetened dietary intake not only elevates calories that, in turn, increase fat and weight, but also may affect glucose homeostasis and insulin sensitivity (Malik et al., 2013; Otero-Losada et al., 2015). Recent studies found that a high intake of sweetened food and beverage elevates the risk of insulin resistance, which consequently decreases hepatic insulin sensitivity and triggers the onset of type 2 diabetes (Bhupathiraju et al., 2013; Maki et al., 2015; Sakurai et al., 2014). The global rise of diabetes is both directly and indirectly associ- ated with being overweight or obese (Fagherazzi et al., 2013; Lim et al., 2016). It is reported that sugar-sweetened beverage intake can increase the incidence of type 2 diabetes (De Koning et al., 2011; Sakurai et al., 2014). It has been revealed that long-term consumption of sweeteners induces glucose intoleratnce, which is specifically linked to type 2 diabetes through fat accumualtion and alteration of gut microbiota (Pereira, 2013; Suez et al., 2014). In addition, diabetics have been shown to have a greater threshold for detecting the sweetness of sugar compared with healthy control subjects, suggesting diabetics increase their sugar intake in order to perceive the same sweet taste-sensing as the healthly control, which in turn, elevates the glucose concentrations in diabetics (Fábián et al, 2015; Neiers et al., 2016). Consequently, numerous studies have been conducted to identify and develop new carbohydrates, sugar alternatives or sweet taste modulators to reduce the risk of obesity and diabetes induced by sweeteners.

Consumers are more interested in new sweet-tasting substances with low- or no-calories to moderate their sugar and energy consumption while still preserving the sweet taste. Numerous non-nutritive sweeteners with a high intensity of sweetness are approved and widely used in industries, such as acesulfame potassium (acesulfame-K). advantame, aspartame, neotame, saccharin and sucralose (U.S. Food and Drug Adminstration, 2018). However, even consumption of low- or no-calorie sweeteners, the roles in reduction effects are limited and controversial (Lohner et al., 2017). A number of studies have shown that long-term use of low- or no-calorie sweetener containing foods may not effectively control weight, because they may not elicit complete energy compensation, but instead, stimulate appetite and promote energy intake, leading to weight gain and obesity (Löfvenborg et al., 2016). Some cohort studies have suggested that chronic use of non-nutritive sweeteners is associated with weight gain, and increasing the obesity risk while deteriorating glucose tolerance (Palmnäs et al., 2014; Pearlman et al., 2019).

\section{Sweeteners}

\subsection{Sugar and sugar alcohols}

Sugars, the most common sweet-tasting natural products known today, are historically used to produce the desirable sweet taste attribute, as well as providing energy to mammals. Since the isolation of sucrose from sugarcane juice, there has been much dietary development and application for daily sugar consumption. Sugars occur naturally in the tissues of various plants, as well as in animal-based products, such as in milk. These soluble carbohydrates are mostly comprised of monosaccharides (i.e. glucose and fructose) and disaccharides (i.e. sucrose, maltose and lactose). Sucrose, one of the most common disaccharides, is used as "table sugar" and as a sensory reference to evaluate the sweetness of other substances. With the exception of glucose and fructose, some other uncommon, natural monosaccharide sugars, including D-allulose (D-psicose), D-tagatose, D-sorbose and D-allose, can elicit a sweet sensation but provide few calories. These are found with small quantities in natural products. D-allulose and D-tagatose are recognized as generally recognized as safe (GRAS) by the U.S. Food and Drug Administration (FDA) and they have been reported to have an anti-obesity effect by decreasing body weight and abdominal fat, and regulating glucose and insulin secretion (Mooradian, Smith, \& Tokuda, 2017; Shintani et al., 2017). In addition, some oligosaccharides, small size molecular polymers with two to ten monosaccharides, are used as prebiotics in foods and provide a 
sweet taste when they are solubilized in water (Belloir et al., 2017).

Sugar alcohols, also known as polyols or polyhydric alcohol, are sweet-tasting carbohydrates that can be obtained from their corresponding aldose sugar. Sugar alcohols are also naturally present in fruits, vegetable or mushroom in a small quantity, and regulated as either GRAS or as food additives (Grembecka, 2015). The most common sugar alcohols include erythritol, isomalt, lactitol, maltitol, mannitol, sorbitol and xylitol. These can induce a relatively low sweetness compared with sucrose while interacting with the sweet taste receptors but avoiding off-taste and producing low calorie. Normally sugar alcohols can combine with other sweeteners to produce a sugar-like flavor in the mouth, and probably mask the off-taste of other sweeteners. It has been reported that most sugar alcohols can prevent tooth decay and act as prebiotics to promote the growth of the beneficial bacteria in the gut (Belloir et al., 2017). They may also promote gut hormone release and have been used as a sugar alternative for people with diabetes (Overduin et al., 2016).

\subsection{Artificial and natural sweeteners}

Due to the concerns related to health problems resulting from overconsumption of sugars, there is a dramatic increase in the demand for low-or zero-calorie sweet-tasting substances. Many natural and artificial sweeteners and sweet-tasting proteins with low-calories have been discovered and used as sugar alternatives to limit caloric intake. Such sweeteners possess a relatively high potency to render sweetness compared with sugar while ingesting fewer calories (Lertrit et al., 2018).

Several artificial sweeteners have been approved as substitutes in either the US or in the EU (aspartame, acesulfame-K, neotame, sucralose and saccharin) (Grembecka, 2015; Steinert et al., 2011). Most of these sweeteners are much sweeter than sucrose and contain low or no calories, which may not affect glucose levels in the body. Saccharin is the oldest artificial sweetener and it possesses 200 to 700 times sweetness than sucrose. Aspartame is more than 200 times sweeter than sucrose, whereas acesulfame-K and sucralose are 300 times and 600 times sweeter than sucrose, respectively. A number of studies have indicated that artificial sweeteners can decrease energy intake from sugar and reduce the weight gain and risk of type 2 diabetes (Raben and Richelsen, 2012). Unfortunately, not all artificial sweeteners are ideal sugar substitutes. Some artificial sweeteners may produce an off-taste, such as bitter, metallic or a licorice-like taste, and slow down or delay sweetness onset (DuBois and Prakash, 2012; Li et al., 2011). Normally, artificial sweeteners are used in binary mixtures to boost the sweetness. However, some may potentially cause adverse effects, such as an augmented risk for obesity, metabolic syndrome and type 2 diabetes (Malik et al., 2013; Pepino, 2015). However, Szimonetta et al. (2017) indicated that, without conclusive evidence, it is hard to establish the correlation between artificial sweeteners and beneficial and harmful effects on health outcomes in the healthy individuals and diabetics (Lohner et al., 2017). The interpretation effect of artificial sweeteners is complicated and confirmative long-term experiments are still needed. Overall, the consumption of artificial sweeteners has increased exponentially over the past several decades (Pearlman et al., 2017).

"Natural sources" of sweeteners have gained considerable attention, resulting in a large number of investigation that are focused on identification and development of the natural source, sweet-tasting substances over the past decades. More than a hundred plants have been reported to taste sweet and the potency of sweetness is much greater than sucrose while still providing low calories. Most natural sweeteners can be classified into the chemical classes of either terpenoids or steroids, or be identified as glycosides (Behrens et al., 2011). The most familiar natural sweetener, Steviol glycosides, is extracted from Stevia Rebaudiana leaves, and include stevioside and rebaudioside A. Stevioside and rebaudioside have a high sweet-tasting potency, at about 300 and 450 times higher than sucrose, respectively. Another natural sugar substitute is mogroside, a cucurbitane-type triterpenoid, from the fruit of Siraitia grosvenorii (Luo Hanguo) (Tu et al., 2017). It provides 250-425 times more sweetness than sucrose, depending on the concentration (Sun et al., 2012; Chiu et al, 2013). Both steviol glycosides and Luo Han Guo fruit extracts mogroside are recognized as GRAS as a non-nutritive sweetener and flavor additive (Mooradian et al., 2017). They have been widely used in the diet of diabetics and obesity patients.

\subsection{Sweet-tasting proteins}

There are six proteins derived from natural plants that can elicit sweetness, including brazzein (54 amino acids, source: African plant Pentadiplandra brazzeana Baillon), pentadin (12 000Da, source: West African Pentadiplandra brazzeana), monellin (two subunits with 42 and 50 amino acids respectively, source: West African shrub Dioscoreophyllum cumminsii), mabinlin (33 and 72 amino acids in $\alpha$ - and $\beta$-chains, respectively, source: Chinese Capparis masaikai), neoculin (curculin) (114 amino acids, source: Malaysian Curculigo latifolia) and thaumatin (207 amino acids, source: West African plant Thaumatococcus daniellii) (Behrens et al., 2011; Iwaniak et al, 2016). A number of studies were conducted to explore similarities of these sweet-tasting proteins, but there is no sequence or structure homology among them (Temussi, 2002). These are high molecular weight polypeptides serval hundred or thousand times sweeter than sucrose, while being low-calorie. Thaumatin is the most intensely sweet protein with 1,660 to 100,000 times more sweetness compared with sucrose on a weight basis, while the sweetness of brazzein, pentadin and mabinlin is 2,000-, 500- and 375-fold more than sucrose. Monellin has two subunits, neither taste sweet, but the undissociated dimer was 3,000-fold "more sweet" compared with sucrose. Neoculin can induce a sweet-taste (550 times over sucrose), while it also can modify sour taste into sweetness (Behrens et al., 2011). Thaumatin has been approved as a sweetener in some European countries and as a GRAS additive in the US (Grembecka, 2015). A sweet-tasting protein is a promising natural sweet source because of the high sweetness potency (Table 1). However, one problem regarding application of sweet-tasting proteins is the difficulty to obtain proteins from their natural sources (Belloir et al., 2017).

\section{Biological basis of sweet taste detection}

\subsection{Sweet taste receptors}

Five basic taste qualities are identified by mammals, i.e. sweet, sour, salty, bitter and umami, to evaluate the quality of foods. Each of them can be sensed by specific taste cells located in the taste buds of the tongue and soft palate. Taste receptor cells are a group of receptors clustered in taste buds, where they play a major role responding to the initial stimuli of different tastes (Kojima et al., 2015). The initial detection begins from the taste molecules binding with taste receptors which then trigger the receptors to release neurotransmitters to that transport where the sensory information is 
Table 1. Selected high-potency sweeteners

\begin{tabular}{|c|c|c|c|}
\hline Compound class & Compound name & Sweetness potency & Reference \\
\hline Disaccharides & Sucrose & 1.0 & DuBois (2016) \\
\hline \multirow[t]{7}{*}{ Monosaccharide } & Glucose & 0.6 & \\
\hline & L-Fructose & 0.6 & \\
\hline & D-Fructose & 1.27 & \\
\hline & D-allulose & 0.7 & Mooradian et al. (2017) \\
\hline & D-tagatose & 0.92 & \\
\hline & D-sorbose & 0.7 & \\
\hline & D-allose & 0.8 & \\
\hline \multirow[t]{7}{*}{ Sugar alcohol } & Erythritol & $0.6-0.8$ & Grembecka (2015) \\
\hline & Isomalt & $0.45-0.65$ & \\
\hline & Lactitol & $0.3-0.4$ & \\
\hline & Maltitol & 0.9 & \\
\hline & Mannitol & $0.5-0.7$ & \\
\hline & Sorbitol & $0.5-0.7$ & \\
\hline & Xylitol & 1.0 & \\
\hline \multirow{12}{*}{$\begin{array}{l}\text { Artificial and natural } \\
\text { sweeteners }\end{array}$} & Acesulfame-K & $100-200$ & \multirow{12}{*}{$\begin{array}{l}\text { Beltrami et al. (2018); } \\
\text { Mooradian et al. (2017) }\end{array}$} \\
\hline & Advantame & $20,000-40,000$ & \\
\hline & Aspartame & 100-200 & \\
\hline & Cyclamate & $\sim 30$ & \\
\hline & Neohesperidin dihydrochalchone & $250-2,000$ & \\
\hline & Neotame & $7,000-13,000$ & \\
\hline & Saccharin & $300-500$ & \\
\hline & Sucralose & $\sim 600$ & \\
\hline & Stevioside & 210 & \\
\hline & rebaudioside & $30-242$ & \\
\hline & Mogroside IV & $233-292$ & \\
\hline & Mogroside V & $250-425$ & \\
\hline \multirow[t]{6}{*}{ Proteins } & Brazzein & $500-2,000$ & \multirow{6}{*}{$\begin{array}{l}\text { Behrens et al. (2011); } \\
\text { Beltrami et al. ( 2018) }\end{array}$} \\
\hline & Curculin (neoculin) & $550-9,000$ & \\
\hline & Mabinlin & 375 & \\
\hline & Monellin & 3,000 & \\
\hline & Pentadin & 500 & \\
\hline & Thaumatin & $1,600-10,000$ & \\
\hline
\end{tabular}

Sweetness potency is given relative to sucrose on a weight comparison

processed and interpreted to brain (Niki et al., 2015). In the mammalian oral cavity, sweet, umami and bitter taste can be sensed by two different G-protein-coupled receptors (GPCRs) families: the Type 1 taste receptors (T1Rs) family, which is mainly involved in the detection of sweet and umami taste molecules; and the Type 2 taste receptors (T2Rs) family, contributed to sense bitter tastelike signaling molecules, while salty and sour tastes are majorly detected by ion-channels (Chao et al., 2016; Running, 2018). It has been well-established that the sweet taste sensation is achieved by two heterodimers of T1Rs subunits, T1R2 (T1Rs, member 2) and T1R3 (T1Rs, member 3).

With further studies into gustation, it has been shown that the sweet taste receptors not only exist in the oral cavity, but also are widely distributed in various non-gustatory organs, including pancreas, colon, stomach, gastrointestinal (GI) tract, brain, bone and adipose tissue, where they have similar sweet taste-sensing functions in the same manner as the tongue, and also contribute to some metabolic regulation in the body, such as glucose homeostasis and 


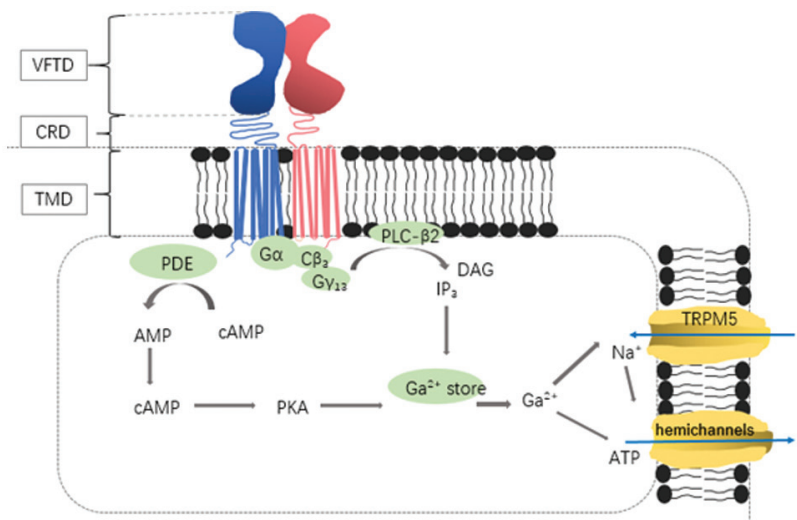

Figure 1. Signal transduction cascade of sweet receptors.

satiety hormone release.

\subsection{Sweetness perception}

The T1R subunits possess 3 principal domains (Figure 1): a large $\mathrm{N}$-terminal extra-cellular domain, often referred as the Venus flytrap domain (VFTD), that provides ligand binding sites to bind sweeteners; a C-terminal seven transmembrane domain (TMD) that contains allosteric binging sites, and a cysteine-rich linker domain (CRD) which connects the VFTD to the TMD (Fernstrom et al., 2012). VDTF has two lobe subdomains which can form an "open" and "closed" conformation like a pocket and has a major area to bind with different sweeteners. The subdomains of VDTF contain orthosteric ligand binding sites to bind and identify various stimuli.

The VFTD of T1R2/T1R3 subunits are the primary binding sites of human sweet taste receptors. Nearly all of the various molecules perceived as sweet-tasting are recognized by the T1R2/T1R3 heterodimers. However, not all sweeteners bind to the same sites on the receptors (Neiers et al., 2016). It has been demonstrated that the sweet tastants bind to the area near the hinge region of the VFTD through a hydrogen bond and trigger the initial closure of the VFTD. Normally, natural sugars (e.g., glucose, fructose, and sucrose) bind to the VFTD of both T1R2 and T1R3. Specifically, VFTD of T1R3 binds sucrose with more affinity than that of T1R2; In contrast, VFTD of T1R2 provides higher affinity to glucose than that of T1R3 (Nie et al., 2005). The artificial sweeteners (e.g., sucralose, aspartame, and neotame) mainly bind to the VFTD of T1R2 (Neiers et al., 2016), whereas, cyclamate and lactisole interact with the TMD of the T1R3 subunit (Xu et al., 2004), and the binding sites of neohesperidin dihydrochalcone (NHDC) overlap with the sweetener cyclamates and the sweetness inhibitor lactisole on T1R3 (Winnig et al., 2007). In addition, detection of sweet proteins such as brazzein, thaumatin and monellin requires interaction with the CRD of human T1R3 (Jiang et al., 2005; Winnig et al., 2007). It is thought that sweet taste enhancers share the same binding sites as large molecular size sweeteners, such as stevioside and aspartame, on the T1R2 VFTD, because the binding for sucrose enhancer overlaps with stevioside and fits in the same upper lobe cavities (Zhang et al., 2010). The T1R3 subunit can be expressed in the taste cells without T1R2, indicating that T1R3 could form a T1R3/T1R3 homodimer whereas T1R1 and T1R2 cannot function by themselves without T1R3 (Belloir et al., 2017; Neiers et al., 2016). It has also been suggested that the T1R3<smiles>CC1NC(N)C2C(C)C(C)C(C)C2N1</smiles>

SE-1

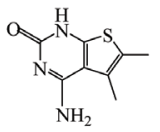

SE-2<smiles>NC1NC(=O)NC2CCCC12</smiles>

SE-3

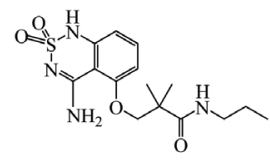

SE-4<smiles></smiles>

DHB<smiles>CC(C(=O)[O-])[N+]1=CC(O)CCC1CO</smiles>

alapyridaine<smiles>CC1CC2NC(=O)NC2C1C</smiles>

ADTP<smiles>CCCNC(=O)C(C)(C)COC1CCC2NC(=O)NC(N)C21</smiles>

ADBT

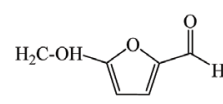

5-hydroxymethyl-2-furaldehyde
Figure 2. Chemical structure of the non-volatile sweetness enhancers. DHB: 2,4-dihydroxybenzoic acid; ADTP: 4-amino-5,6-dimethylthieno(2,3D) pyrimidin-2(1H)-one; ADBT: 3-(4-amino-2, 2-dioxido-1H-2,1,3- benzothiadiazin-5-yloxy)-2,2-dimethyl-N-propylpropanamide.

homodimer acts as a receptor for natural mono- and disaccharides (Kurimoto, 2003; Neiers et al., 2016). Sweetness enhancer molecules are most likely to bind to the area nearest to the open area of the VFTD and help to stabilize the closed conformation of VFTD (Zhang et al., 2010). Sweetness enhancer may improve the binding affinity between the sweeteners and receptors within the VFTD, rather than directly activate the receptors (Beltrami et al., 2018; Guy Servant et al., 2011). It is also proposed that sweetness enhancers can bind receptors at the TMD to enhance activity (Guy Servant et al., 2011)

The activation of taste receptors can induce and increase cytoplasmic $\mathrm{Ca}^{2+}$ and/or cyclic adenosine monophosphate (cAMP) to transmit intracellular signals, leading to perception of sweetness. The binding of T1R2/T1R3 with different stimuli acts with heterotrimeric G-protein gustducin $\alpha, \beta$ and $\gamma$ subunits ( $\alpha$-gustducin, $\mathrm{C} \beta_{3}$ and $\mathrm{G} \gamma_{13}$ ) and subsequently initiates a transduction cascade via phospholipase $C \beta 2$ (PLC- $\beta 2$ ) to generate two messengers, 1,4,5-inositol trisphosphate (IP3) and diacylglycerol (DAG) (Kinnamon, 2012). IP3 interacts with the Type III IP3 receptor (IP3R3) to induce and increase intercellular $\mathrm{Ca}^{2+}$ release, which can gate the transduction channel TRPM5 (a sodium-permeable cation channel) in the membrane ( Laffitte et al., 2014; Young et al., 2009). The $\mathrm{Ca}^{2+}$-activated TRPM5 channels can cause flux of sodium $\left(\mathrm{Na}^{+}\right)$and depolarizaiton of membrane, leading to the release of ATP through pannexin-1 hemichannels (Kinnamon, 2012; Kojima et al., 2014). Additionally, gustducin knockout mice can sense sweet taste, suggesting an additional pathway for sweetness perception. Some studies have indicated that sweet taste receptor may activate a cAMP-dependent pathway producing a longer sensation of sweetness, whereas PLC- $\beta 2$ pathway probably induces a short time sensing (Figure 2) (Behrens et al., 2011; Kinnamon, 2012; Laffitte et al., 2014). $\alpha$-Gustducin can regulate cAMP levels through activation of a phosphodiesterase (PDE), curbing phosphorylation and desensitization of $\mathrm{Ca}^{2+}$ signaling effectors.

Abilities for sweet perception vary among species. For instance, studies show that rodents cannot identify some sweeteners that can be detected by human, such as cyclamate, aspartame, NHDC and sweet-tasting proteins (Winnig et al., 2007). In addition, genetic 
studies have indicated that human tongues are not all alike (Belloir et al., 2017). The genetic variation of taste receptors influences the sense of sweet taste and consumption of sweeteners. For example, the variation of the taste receptor gene of people in West Mexico attributes to a high consumption of carbohydrates (Ramos-Lopez et al., 2016). In addition, there is a correlation between genetic variants in T1R with dietary consumption among Korean populations, which showed genetic variants in T1R1 polymorphisms affected the intake of sweets and vegetables, wherease T1R2 polymorphisms influence consumption of cruciferous vegetables, citrus fruits, fatty and umami food (Choi et al., 2016).

\section{Physiological effect of sweeteners and sweet taste receptors on obesity and diabetes}

It has been suggested that the T1R2/T1R3 sweet taste receptor on the tongue and in different organs not only senses sweet taste, but is also involved in glucose sensing, expression of glucose transporters, gut hormone secretion and maintaining of glucose homeostasis. The dysfunction of sweet taste receptors in the body is correlated with metabolic problems, leading to obesity and diabetes (Belloir et al., 2017; Neiers et al., 2016; Smith et al., 2016). It is proposed that T1R taste receptors could provide novel therapeutic method through modulating sugar absorption and downregulating diet-related disorder to inhibit and attenuate diabetes and obesity.

In the pancreas, insulin released by the $\beta$-cells is proportional to plasma glucose levels and it is especially dependent on the acute change of glucose. The T1R2/T1R3 receptor has been found to be expressed by pancreatic $\beta$-cells, where they can respond to sugar, and are involved in modulating insulin secretion (Kyriazis et al., 2012; Medina et al., 2014). A previous study demonstrated that knockout T1R3 in isolated islets impaired glucose-induced insulin secretion (Kojima et al., 2015), suggesting that sweet taste receptors are involved in insulin secretion. Under normal physiological condition, the glucose molecule interacts with sweet taste receptors in $\beta$-cells to promote insulin release, while high level glucose would reduce the expression of sweet taste receptors in the $\beta$-cells (Belloir et al., 2017; Kyriazis et al., 2014). The diabetic and dietinduced obese individuals have similar decreased effects on the expression of sweet taste receptors, suggesting diabetes and obesity impair sweet taste receptors and alter their function on $\beta$-cells (Kyriazis et al., 2014). With the exception of glucose, there is an additional way in which sweet taste-sensing receptors are involved in the regulation of insulin release. For example, sweet taste receptors on mouse and human $\beta$-cells can detect fructose and stimulate insulin secretion (Kyriazis et al., 2014). In addition, the inhibitors of sweet taste receptors, such as gurmarin, can significantly curb glucose-induced insulin release from $\beta$-cells (Kojima et al., 2015).

The regulation of insulin secretion by sweet taste receptors not only through glucose interaction, but also via indirect modulation of the glucose-related hormone in the gastrointestinal (GI) tract when glucose is ingested. It is known that T1R receptors and the taste $\mathrm{G}$ protein gustducin are expressed in enteroendocrine $\mathrm{L}$ - and $\mathrm{K}$-cells in the GI tract, where they not only function as a sweet taste sensors, but also induce hormone secretion to maintain glucose homeostasis (Greenfield and Chisholm, 2013; Nomura and Kawahara, 2015; Shirazi-Beechey et al., 2014). Glucagon-like peptide-1 (GLP-1) and glucose-dependent insulinotropic peptide (GIP) are the major incretin hormones which respond to the transmission of sweet taste signals and promote insulin release, whereas Glucagon-like peptide-2 (GLP-2) increases intestinal growth and glucose absorption (Feng et al., 2017; Smith et al., 2016). Chol- ecystokinin (CCK) and peptide tyrosine-tyrosine (PYY) play a variety of roles in digestive processes, including reduction of food consumption and increasing satiety (Daly et al., 2013; Gerspach et al., 2011). It has been demonstrated that oral ingestion of glucose and sucrose or directly infusing sugars, including D-isoforms of glucose, galactose and fructose and non-metabolizable analogues of glucose, 3-O-methyl-glucose and a-methyl-glucose, into intestinal lumen can activate with T1R2/T1R3 inducing and increasing GLP-1 and GIP secretion (Dyer et al., 2007; Sakurai et al., 2012; Steinert et al., 2011). Brown et al. (2012) confirmed that diet soda sweetened with sucralose and acesulfame-K with glucose can increase GLP-1 secretion in healthy subjects. The consumption of sugar alcohol does not elicit an elevation in blood glucose and insulin secretion, but it can affect gut hormones (Grembecka, 2015). A recent study demonstrated that xylitol and erythritol intake can stimulate GLP-1 and CCK release in the gut of both lean and obese subjects (Wölnerhanssen et al., 2016). Overduin et al. (2016) reported that erythritol has a similar effect on trigger and control GLP-1 and PYY release in lean (not obese) subjects compared with sucrose. Similar in vitro results indicated that both sugars and artificial sweeteners (e.g. aspartame and sucralose) stimulate and increase the secretion of GLP-1 and GIP (Jang et al., 2007; Ma et al., 2009; Malaisse, 2014; Sakurai et al., 2012). However, the underlying mechanism of sweeteners induced gastrointestinal peptide secretion remains unclear. Additionally, the gut-expressed taste receptor involved in regulating expression of sodium-glucose is cotransporter-1 (SGLT-1), which is the primary transporter of sugars in the intestinal lumen (Margolskee et al., 2007; Stearns et al., 2010). Dietary sugar and artificial sweeteners upregulate SGLT-1 mRNA and protein expression and glucose uptake in mice but not in T1R3 knockout or gustducin present mice (Dyer et al., 2007; Margolskee et al., 2007). In type 2 diabetics, SGLT-1 and the ability of glucose absorption may be improved intestinally, whereas GLP-1 and GIP release is decreased (Young et al., 2013); this may be due to gut taste receptor expression decreasing in diabetic subjects with elevated blood glucose concentrations (Young et al., 2009).

In the body, the sensitivity of sweet taste receptors would also be influenced by some metabolic hormones, such as leptin and endocannabinoids (Yoshida et al., 2013). Leptin is an anorexigenic mediator which can selectively suppress sweet taste receptor responses to sweetness, whereas it did not affect other taste sensations (Stearns et al., 2010). Leptin can elevate glucose perceptive thresholds, suggesting a high concentration of leptin probably triggers high a BMI value. Endocannabinoids, such as anandamide [N-arachidonoylethanolamine (AEA)] and 2-arachidonoyl glycerol (2-AG), can act as a sweet taste enhancer, showing an adverse effect of leptin. It is reported that administration of AEA or 2-AG can elevate responses to sweeteners, whereas they do not affect the sensation of other taste qualities ( Yoshida et al., 2010).

\section{Sweet taste enhancer}

A new way to attenuate the health problems caused by sweetness is positive allosteric modulations (PAMs) of sweet taste receptor, leading to the discovery and identification of sweet taste enhancement. The enhancing molecular does not induce sweetness sensations on their own but potentiate the sweet sense of other sweeteners through binding with sweet taste receptors. These enhancers primarily bind to the TMD of receptors to enhance receptor activity, as well as increase the binding affinity between sweeteners and receptors within the VFTD. The application of sweet taste en- 
hancers is very important to the food industry, because it can help decrease excessive calorie intake through reduction of the sugar or sugar alternatives, and at the same time, provide a desirable sweet taste quality. Certain compounds have been identified and claim to have the ability to improve sweet taste of some sugar, but the enhancement is limited to a selective effect on varying sugars.

\subsection{The artificial sweet taste enhancer}

The first example of a sweetness enhancer, named as SE-1, was identified and reported by the company Senomyx Incorporation (San Diego, CA, USA) in collaboration with Coca-Cola company (Guy Servant et al., 2011). It is reported that SE-1 can selectively increase the sweet taste intensity of sucralose by $>20$ times, which can reduce the concentration of sucralose by $50 \%$ while maintaining sweetness intensity (Servant et al., 2010). Subsequently, other sweet enhancers, including SE-2, SE-3 and SE-4 were discovered by Senomyx. They have the ability to selectively improve the sweetness of different sweeteners, without inducing a taste by themselves. SE-2 can reduce the concentration of sucralose more than $50 \%$ while preserving sweetness intensity, whereas SE-3 provides a $33 \%$ decrease in the sucrose concentration while retaining the same sweetness intensity ( Servant et al., 2010; Guy Servant et al., 2012). Cell-based assays indicate these are specific to the human sweet taste receptors and have no effect on rat receptors (Zhang et al., 2010). A certain number of synthetic PAM compounds with the capacity to increase sweet taste have been patented by Senomyx Inc. as sweetness modifiers (Tachdjian et al., 2013, 2010). In addition, more sweetness enhancers were reported by Mnique et al., (2018), including 2,4-dihydroxybenzoic acid (DHB), 4-amino-5,6-dimethylthieno(2,3-D) pyrimidin-2(1H)-one (ADTP) and 3-(4-amino-2, 2-dioxido-1H-2,1,3- benzothiadiazin-5-yloxy)-2,2dimethyl-N-propylpropanamide (ADBT). DHB, ADTP and ADBT are artificial flavor compounds, which can enhance the sweetness for selective sweeteners. All of these have been approved as artificial flavors by Flavor and Extract Manufacturers Association (FEMA). DHB has been reported to have the ability to improve the sweet taste of aspartame from 0.1 to 4.0 times, while Holland Sweetener Company indicated DHB does not have this effect on aspartame, but can improve the sweetness of $6 \%$ sucrose to more than $8 \%$ sucrose $(>33 \%$ enhancement), whereas it does not taste sweet by itself (DuBois and Prakash, 2012). ADTP has been reported to selectively amplify the sweet taste intensity of sucralose, which can improve the sweetness of a sucralose solution six to eight times, whereas increase the sweetness intensity of sucrose with two to twelve times at certain concentrations (DuBois and Prakash, 2012; Guy Servant et al., 2012). ADBT was discovered as a sucrose-selective enhancer to increase $6 \%$ sucrose level to the equivalent of a $10.6 \%$ sucrose level (i.e., 1.8 -fold) at $8.8 \mathrm{mg} / \mathrm{L}$ by human sensory panelists (DuBois and Prakash, 2012).

\subsection{Food-processed sweet taste enhancer}

During the food process, many reactions, such as Maillard reaction, produce flavor compounds that improve or impair the quality of products. Two food processed compounds reported by the Hofmann group, have the potential to enhance sweet taste (Ottinger and Hofmann, 2003; Soldo et al., 2003) N-(1-carboxyethyl)-6(hydroxymethyl) yridinium-3-ol inner salt, commonly known as pyridinium betaine or alapyridaine, is a product of Maillard reaction, which was found and isolated from heated hexoses and Lalanine mixtures, as well as in beef broth. It has been demonstrated alapyridaine significantly enhances the taste of sweet, umami and salt sensations (Ottinger and Hofmann, 2003). With the presence of alapyridaine, the sweet taste limitation of glucose and sucrose, as well as the umami taste of monosodium L-glutamate (MSG) and guanosine-5'-monophosphate (GMP) were remarkably reduced (Soldo et al., 2003). Another compound, 5-acetoxymethyl-2-furaldehyde, was found in vinegar of Modena (TBV). It has been indicated to improve the sweet taste quality of TBV. The presence of 5-acetoxymethyl-2-furaldehyde triggered a long-lasting sweetness intensity of a $4 \%$ sucrose solution, while alone, it does not exhibit a sweet taste in human sensory tests (Hillmann et al., 2012).

\subsection{Natural sweet taste enhancer}

Miracle fruit (Synsepalum dulcificum) has been recognized as a sweetness enhancer, because by itself, it has no taste, but it can convert a sour taste into sweet (Iwaniak et al., 2016). The active compound in miracle fruit is miraculin, a glycoprotein, which possesses sweetness-inducing activity. Miraculin does not taste sweet by itself, but does bind with the sweet taste receptor to simulate an effect of 400,000-fold sweeter than sucrose on a mole basis (Kurihara and Beidler, 1968). Miraculin binds mostly to the amino-terminal domain of T1R2, activating the receptor in response to a low $\mathrm{pH}$, that then produces a sweet taste. The sweetness-inducing effect of miraculin is $\mathrm{pH}$-dependent. Weak acids produce a higher intensity of sweet taste compared with strong acids (Koizumi et al., 2011). In a neutral pH, miraculin binds with T1R2 in an inactive form, resulting in no sweet taste response. However, under acidic conditions, the extracellular region of T1R2 and miraculin are protonated in the presence of acid $(\mathrm{H}+)$, causing activation of an intracellular signaling cascade, and thereby evoking a sweet sensation. Furthermore, the mechanisms of how miraculin modifies the acidification into a sweet sensation through activation of the sweet taste receptor is still unclear. The induction of a sweet taste by miraculin in acid is specific to human receptors. It does not occur in rodents (Sanematsu et al., 2016). Miracle fruit has a great potential future to both modify and mask undesirable tastes, as well as elicit sweetness.

\subsection{Volatile compounds}

Most of the sweet-taste substances are non-volatiles. However, some volatiles or aromas can interact with sweet taste perception to enhance sweetness perception. Multiple volatile compounds have been reported to make an important contribution to the sweetness of fruits. Studies have shown that the presence of some odorants, including strawberry, almond, caramel, coffee, lemon, peach and vanilla aromas, can increase the perception of the sweetness intensity of several sweeteners, such as sucrose, fructose, aspartame and saccharine (Valentin et al., 2006). Additionally, in a previous study, maltol (2-methyl-3-hydroxy-pyran-4-one) was reported to have the ability to increase the sweet taste of sugar (Methven, 2012). Maltol has a candy-like odor, which can be used to impart a sweet aroma. In a recent study, isoamyl acetate, a volatile ester, was found to increase the sweetness intensity of sucrose by pulsatile delivery (Burseg et al., 2010). In addition, three phthalides, sedanenolide, 3-n-butylphthalide and sedanolide, were identified in chicken broth with a celery fraction to have the ability to enhance the perception of the sensation of both umami and sweet whereas they have no taste characteristics on their own (Kurobayashi et al., 2008). Similar volatile compounds such as 4-hydroxy-2(or 5)-ethyl-5(or 2)-methyl-3(2H)-furanone, 2-hydroxy-3-methyl- 
2-cyclopenten-1-one and 3-hydroxy-4,5-dimethyl-2(5H)-furanone have also been found to enhance sweet perception (Methven, 2012). In fact, volatile-induce sweet taste enhancement is not new. However, few studies have investigated how volatile might alter the perception or interaction with various sweeteners. One issue with applying volatile compounds to enhance sweet taste is that their own characteristic aroma may be inappropriate for the food products targeted for enhancement, even though most volatiles are tasteless. Aroma compounds will induce new aroma with different foods. Another problem is the low solubility of aroma sweetness enhancers which brings challenge to food manufacture. Additionally, volatile ability of most aromas results in an instability of sweet taste because these compounds easily volatilize.

\section{Conclusion}

There is an increasing desire to reduce calorie intake from sugaradded foods while maintaining a desirable sweet sensation. Nonnutritive sweeteners have been widely applied to foods to limit the calories ingested from sugar, but the off-taste and aftertaste have limited the application of such sweeteners. Orally, T1R2/ T1R3 plays an important role perceiving the sweetness of various stimuli that are involved in dietary food consumption and caloric intake. In organs, other than those that are sweet taste-sensing, T1R2/T1R3 has been shown to contribute to many metabolic regulations, including glucose homeostasis, satiety hormone release and regulation of insulin secretion. Some metabolic hormones, such as leptin and endocannabinoids, also act as a modulator of sweetness to regulate sugar intake. There is an association between sweet taste receptors and many health problems, including obesity and diabetes. Therefore, the sweet receptor is a potential molecular therapeutic target to prevent obesity and treat diabetes.

With the discovery of sweet taste receptors, sweet taste enhancement implies a possible strategy for maintaining and potentiating a sugar taste, while limiting calories. Sweet taste enhancers offer a bright future for regulating caloric intake by limiting sugar levels in food. However, few examples of natural enhancers have been identified. Studies have demonstrated many aromas that have the ability to improve sweet taste intensity of some sweeteners, but there are limited studies into the interaction between aroma and sweet taste enhancement. Further examination of the effect of odor-induced sweet taste enhancement can extend the possibility of natural sweetness enhancers in foods. However, producing foods with aroma sweetness enhancers is challenging, due to low solubility. Non-volatile sweetness enhancers are more stable than aromatic ones, while still increasing a desired sweet taste. Even though there are many synthetic sweetness enhancers both identified and studied, they are not readily used by the industries, due to regulatory limitations and consumer demands for clean label and natural products. Therefore, identification of new, natural sweetness enhancers is crucial and may drive a better application of enhancers by the food industry.

\section{References}

Adamski-Werner, S.L., Darmohusodo, V., Tachdjian, C., Karanewsky, D.S., and Senomyx Inc. (2015). Sweet flavor modifier. U.S. Patent $9,000,151$.

Ariyasu, T., Matsumoto, S., Kyono, F., Hanaya, T., Arai, S., Ikeda, M., and Kurimoto, M. (2003). Taste receptor T1R3 is an essential molecule for the cellular recognition of the disaccharide trehalose. In Vitro Cell. Dev. Biol. -Animal 39(1-2): 80-88.
Bai-Shen Sun, B.S., Chen, Y.P., Wang, Y.B., Tang, S.W., Pan, F.Y., Li, Z., and Sung, C.K. (2012). Anti-obesity effects of mogrosides extracted from the fruits of Siraitia grosvenorii (Cucurbitaceae). Afr. J. Pharm. Pharmacol. 6(20): 1492-1501.

Behrens, M., Meyerhof, W., Hellfritsch, C., and Hofmann, T. (2011). Sweet and umami taste: Natural products, their chemosensory targets, and beyond. Angew. Chem.-Int. Edit. 50(10): 2220-2242.

Belloir, C., Neiers, F., and Briand, L. (2017). Sweeteners and sweetness enhancers. Curr. Opin. Clin. Nutr. Metab. Care. 20(4): 279-285.

Bhupathiraju, S.N., Pan, A., Malik, V.S., Manson, J.E., Willett, W.C., van Dam, R.M., and Hu, F.B. (2013). Caffeinated and caffeine-free beverages and risk of type 2 diabetes. Am. J. Clin. Nutr. 97(1): 155-166.

Burseg, K.M.M., Camacho, S., Knoop, J., and Bult, J.H.F. (2010). Sweet taste intensity is enhanced by temporal fluctuation of aroma and taste, and depends on phase shift. Physiol. Behav. 101(5): 726-730.

Beltrami, M.C., DÖRING, T., and Lindner, J.D.D. (2018). Sweeteners and sweet taste enhancers in the food industry. Food Sci. Technol. 38(2): 181-187.

Chao, D.H.M., Argmann, C., Van Eijk, M., Boot, R.G., Ottenhoff, R., Van Roomen, C., Foppen, E., Siljee, J.E., Unmehopa, U.A., Kalsbeek, A. and Aerts, J.M.F.G. (2016). Impact of obesity on taste receptor expression in extra-oral tissues: Emphasis on hypothalamus and brainstem. Scientific Reports. 6(1): 29094.

Chia, C.W., Shardell, M., Tanaka, T., Liu, D.D., Gravenstein, K.S., Simonsick, E.M., Egan, J.M., and Ferrucci, L. (2016). Chronic Low-Calorie Sweetener Use and Risk of Abdominal Obesity among Older Adults: A Cohort Study. PLoS ONE. 11(11): e0167241.

Chiu, C.H., Wang, R., Lee, C.C., Lo, Y.C., and Lu, T.J. (2013). Biotransformation of mogrosides from Siraitia grosvenorii swingle by Saccharomyces cerevisiae. J. Agric. Food Chem. 61(29): 7127-7134.

Choi, J.H., Lee, J., Choi, I.J., Kim, Y.W., Ryu, K.W., and Kim, J. (2016). Variations in TASIR taste receptor gene family modify food intake and gastric cancer risk in a Korean population. Mol. Nutr. Food Res. 60(11): 2433-2445.

Daly, K., Al-Rammahi, M., Moran, A., Marcello, M., Ninomiya, Y., and Shirazi-Beechey, S.P. (2013). Sensing of amino acids by the gut-expressed taste receptor T1R1-T1R3 stimulates CCK secretion. Am. J. Physiol. Gastrointest. Liver Physiol. 304(3): G271-G282.

De Koning, L., Malik, V.S., Rimm, E.B., Willett, W.C., and Hu, F.B. (2011). Sugar-sweetened and artificially sweetened beverage consumption and risk of type 2 diabetes in men. Am. J. Clin. Nutr. 93(6): 13211327.

DuBois, G.E. (2016). Molecular mechanism of sweetness sensation. Physiol. Behav. 164: 453-463.

DuBois, G.E., and Prakash, I. (2012). Non-Caloric Sweeteners, Sweetness Modulators, and Sweetener Enhancers. Annu. Rev. Food Sci. Technol. 3(1): 353-380.

Dyer, J., Daly, K., Salmon, K.S.H., Arora, D.K., Kokrashvili, Z., Margolskee, R.F., and Shirazi-Beechey, S.P. (2007). Intestinal glucose sensing and regulation of intestinal glucose absorption. Biochem. Soc. Trans. 35(Pt 5): 1191-1194.

Fábián, T.K., Beck, A., Fejérdy, P., Hermann, P., and Fábián, G. (2015). Molecular mechanisms of taste recognition: Considerations about the role of saliva. Int. J. Mol. Sci. 16(3): 5945-5974.

Fagherazzi, G., Vilier, A., Saes Sartorelli, D., Lajous, M., Balkau, B., and Clavel-Chapelon, F. (2013). Consumption of artificially and sugar-sweetened beverages and incident type 2 diabetes in the Etude Epidémiologique auprès des femmes de la Mutuelle Générale de l'Education Nationale-European Prospective Investigation into Cancer and Nutrition cohort. Am. J. Clin. Nutr. 97(3): 517-523.

Feng, R., Qian, C., Liu, Q., Jin, Y., Liu, L., Li, S., Liao, Y., Zhou, H., Liu, W., Rayner, C.K., and Ma, J. (2017). Expression of sweet taste receptor and gut hormone secretion in modelled type 2 diabetes. Gen. Comp. Endocrinol. 252: 142-149.

Zhang, F., Klebansky, B., Fine, R.M., Liu, H., Xu, H., Servant, G., Zoller, M., Tachdjian, C., and Li, X. (2010). Molecular mechanism of the sweet taste enhancer. PNAS 107(10): 4752-4757.

Fernstrom, J.D., Munger, S.D., Sclafani, A., De Araujo, I.E., Roberts, A., and Molinary, S. (2012). Mechanisms for Sweetness. J. Nutr. 142(6): 1134S-1141S.

Fowler, S.P., Williams, K., Resendez, R.G., Hunt, K.J., Hazuda, H.P., and 
Stern, M.P. (2008). Fueling the Obesity Epidemic? Artificially Sweetened Beverage Use and Long-term Weight Gain. Obesity. 16(8): 1894-1900.

Gerspach, A.C., Steinert, R.E., Schonenberger, L., Graber-Maier, A., and Beglinger, C. (2011). The role of the gut sweet taste receptor in regulating GLP-1, PYY, and CCK release in humans. A.J.P. Endocrinol. Metab.301(2): E317-E325

Greenfield, J.R., and Chisholm, D.J. (2013). How sweet it is: Intestinal sweet taste receptors in type 2 diabetes. Diabetes. 62(10): 3336-3337.

Grembecka, M. (2015). Sugar alcohols-their role in the modern world of sweeteners: a review. Eur. Food Res. Technol. 241(1): 1-14.

Hillmann, H., Mattes, J., Brockhoff, A., Dunkel, A., Meyerhof, W., and Hofmann, T. (2012). Sensomics analysis of taste compounds in balsamic vinegar and discovery of 5-acetoxymethyl-2-furaldehyde as a novel sweet taste modulator. J. Agric. Food Chem. 60(40): 9974-9990.

Horio, N., Jyotaki, M., Yoshida, R., Sanematsu, K., Shigemura, N., and Ninomiya, Y. (2010). New Frontiers in Gut Nutrient Sensor Research: Nutrient Sensors in the Gastrointestinal Tract: Modulation of Sweet Taste Sensitivity by Leptin. J. Pharmacol. Sci. 112(1): 8-12.

Huang, C., Huang, J., Tian, Y., Yang, X., and Gu, D. (2014). Sugar sweetened beverages consumption and risk of coronary heart disease: A metaanalysis of prospective studies. Atherosclerosis 234(1): 11-16.

Iwaniak, A., Minkiewicz, P., Darewicz, M., and Hrynkiewicz, M. (2016). Food protein-originating peptides as tastants - Physiological, technological, sensory, and bioinformatic approaches. Food Res. Int. 89: 27-38

Jang, H.J., Kokrashvili, Z., Theodorakis, M.J., Carlson, O.D., Kim, B.J., Zhou, J., Kim, H.H., Xu, X., Chan, S.L., Juhaszova, M., and Egan, J.M. (2007) Gut-expressed gustducin and taste receptors regulate secretion of glucagon-like peptide-1. Proc. Natl. Acad. Sci. U. S. A. 104(38): 15069-15074.

Jiang, P., Cui, M., Zhao, B., Snyder, L.A., Benard, L.M.J., Osman, R., Max, M., and Margolskee, R.F. (2005). Identification of the cyclamate interaction site within the transmembrane domain of the human sweet taste receptor subunit T1R3. J. Biol. Chem. 280(40): 34296-34305.

Kinnamon, S.C. (2012). Taste receptor signalling - from tongues to lungs. Acta Physiol. 204(2): 158-168.

Koizumi, A., Tsuchiya, A., Nakajima, K.I., Ito, K., Terada, T., Shimizu-lbuka, A., Briand, L., Asakura, T., Misaka, T., and Abe, K. (2011). Human sweet taste receptor mediates acid-induced sweetness of miraculin. Proc. Natl. Acad. Sci. 108(40): 16819-16824.

Kojima, I., Nakagawa, Y., Hamano, K., Medina, J., Li, L., and Nagasawa, M. (2015). Glucose-Sensing Receptor T1R3: A New Signaling Receptor Activated by Glucose in Pancreatic $\beta$-Cells. Biol. Pharm. Bull 38(5): 674-679.

Kojima, I., Nakagawa, Y., Ohtsu, Y., Medina, A., and Nagasawa, M. (2014). Sweet Taste-Sensing Receptors Expressed in Pancreatic $\beta$-Cells: Sweet Molecules Act as Biased Agonists. Endocrinol. Metab. 29(1): 12.

Kurihara, K., and Beidler, L.M. (1968). Taste-modifying protein from miracle fruit. Science 161(3847): 1241-1243.

Kurobayashi, Y., Katsumi, Y., Fujita, A., Morimitsu, Y., and Kubota, K. (2008). Flavor enhancement of chicken broth from boiled celery constituents. J. Agric. Food Chem. 56(2): 512-516.

Kyriazis, G.A., Smith, K.R., Tyrberg, B., Hussain, T., and Pratley, R.E. (2014). Sweet taste receptors regulate basal insulin secretion and contribute to compensatory insulin hypersecretion during the development of diabetes in male mice. Endocrinology 155(6): 2112-2121.

Kyriazis, G.A., Soundarapandian, M.M., and Tyrberg, B. (2012). Sweet taste receptor signaling in beta cells mediates fructose-induced potentiation of glucose-stimulated insulin secretion. Proc. Natl. Acad. Sci. U. S. A. 109(8): E524-32.

Laffitte, A., Neiers, F., and Briand, L. (2014). Functional roles of the sweet taste receptor in oral and extraoral tissues. Curr. Opin. Clin. Nutr. Metab. Care. 17(4): 379-385.

Lertrit, A., Srimachai, S., Saetung, S., Chanprasertyothin, S., Chailurkit, L., Areevut, C., Katekao, P., Ongphiphadhanakul, B., and Sriphrapradang, C. (2018). Effects of sucralose on insulin and glucagon-like peptide-1 secretion in healthy subjects: a randomized, double-blind, placebocontrolled trial. Nutrition. 55-56: 125-130.

Li, X., Servant, G., and Tachdjian, C. (2011). The discovery and mechanism of sweet taste enhancers. Biomol. Concepts. 2(4): 327-332.

Lim, E., Lim, J.Y., Kim, E., Kim, Y.S., Shin, J.H., Seok, P.R., Jung, S., Yoo, S., and Kim, Y. (2016). Xylobiose, an alternative sweetener, ameliorates diabetes-related metabolic changes by regulating hepatic lipogenesis and miR-122a/33a in db/db Mice. Nutrients 8(12): 791.

Löfvenborg, J.E., Andersson, T., Carlsson, P.O., Dorkhan, M., Groop, L., Martinell, M., Tuomi, T., Wolk, A., and Carlsson, S. (2016). Sweetened beverage intake and risk of latent autoimmune diabetes in adults (LADA) and type 2 diabetes. Eur. J. Endocrinol. 175(6): 605-614.

Lohner, S., Toews, I., and Meerpohl, J.J. (2017). Health outcomes of nonnutritive sweeteners: analysis of the research landscape. Nutr. J. 16(1): 55.

Ma, J., Bellon, M., Wishart, J.M., Young, R., Blackshaw, L.A., Jones, K.L., Horowitz, M., and Rayner, C.K. (2009). Effect of the artificial sweetener, sucralose, on gastric emptying and incretin hormone release in healthy subjects. A.J.P. Gastrointest. Liver Physiol. 296(4): G735G739.

Mace, O.J., Affleck, J., Patel, N., and Kellett, G.L. (2007). Sweet taste receptors in rat small intestine stimulate glucose absorption through apical GLUT2. J. Physiol. 582(1): 379-392.

Maki, K.C., Nieman, K.M., Schild, A.L., Kaden, V.N., Lawless, A.L., Kelley K.M., and Rains, T.M. (2015). Sugar-Sweetened Product Consumption Alters Glucose Homeostasis Compared with Dairy Product Consumption in Men and Women at Risk of Type 2 Diabetes Mellitus. J. Nutr. 145(3): 459-466.

Malaisse, W.J. (2014). Insulin release: The receptor hypothesis. Diabetologia $57(7):$ 1287-1290

Malik, V.S., Pan, A., Willett, W.C., and Hu, F.B. (2013). Sugar-sweetened beverages and weight gain in children and adults: A systematic review and meta-analysis. Am. J. Clin. Nutr. 98(4): 1084-1102.

Margolskee, R.F., Dyer, J., Kokrashvili, Z., Salmon, K.S.H., llegems, E., Daly, K., Maillet, E., Ninomiya, Y., Mosinger, B., and Shirazi-Beechey, S.P. (2007). T1R3 and gustducin in gut sense sugars to regulate expression of Na+-glucose cotransporter 1. Proc. Natl. Acad. Sci. 104(38) $15075-15080$.

Medina, A., Nakagawa, Y., Ma, J., Li, L., Hamano, K., Akimoto, T., Ninomiya, Y., and Kojima, I. (2014). Expression of the glucose-sensing recepto T1R3 in pancreatic islet: changes in the expression levels in various nutritional and metabolic states. Endocr. J. 61(8): 797-805.

Baines, D., and Seal, R. (2012). Natural food additives, ingrediets and flavourings. Elservier, pp. 76-99.

Mooradian, A.D., Smith, M., and Tokuda, M. (2017). The role of artificial and natural sweeteners in reducing the consumption of table sugar: A narrative review. Clin. Nutr. ESPEN. 18: 1-8.

Neiers, F., Canivenc-Lavier, M.C., and Briand, L. (2016). What Does Diabetes "Taste" Like? Curr. Diab. Rep. 16(6): 49.

Nie, Y., Vigues, S., Hobbs, J.R., Conn, G.L., and Munger, S.D. (2005). Distinct contributions of T1R2 and T1R3 taste receptor subunits to the detection of sweet stimuli. Curr. Biol. 15(21): 1948-1952.

Niki, M., Jyotaki, M., Yoshida, R., Yasumatsu, K., Shigemura, N., DiPatrizio, N.V., Piomelli, D., and Ninomiya, Y. (2015). Modulation of sweet taste sensitivities by endogenous leptin and endocannabinoids in mice. J. Physiol. 593(11): 2527-2545.

Nomura, M., and Kawahara, Y. (2015). Role of the Sweet Taste Receptor in Glucose Metabolism: No Sweets for Diabetes? Yakugaku Zasshi. 135(6): 763-767.

Otero-Losada, M., Cao, G., González, J., Muller, A., Ottaviano, G., Lillig, C., Capani, F., Ambrosio, G., and Milei, J. (2015). Functional and Morphological Changes in Endocrine Pancreas following Cola Drink Consumption in Rats. PLoS ONE 10(3): e0118700.

Ottinger, H., and Hofmann, T. (2003). Identification of the Taste Enhancer Alapyridaine in Beef Broth and Evaluation of Its Sensory Impact by Taste Reconstitution Experiments. J. Agric. Food Chem. 51(23): 6791-6796.

Overduin, J., Collet, T.H., Medic, N., Henning, E., Keogh, J.M., Forsyth, F. Stephenson, C., Kanning, M.W., Ruijschop, R.M.A.J., Farooqi, I.S., and Van der Klaauw, A.A. (2016). Failure of sucrose replacement with the non-nutritive sweetener erythritol to alter GLP-1 or PYY release or test meal size in lean or obese people. Appetite. 107: 596-603.

Palmnäs, M.S.A., Cowan, T.E., Bomhof, M.R., Su, J., Reimer, R.A., Vogel, H.J., Hittel, D.S., and Shearer, J. (2014). Low-Dose Aspartame Con- 
sumption Differentially Affects Gut Microbiota-Host Metabolic Interactions in the Diet-Induced Obese Rat. PLoS ONE 9(10): e109841.

Pearlman, M., and Akpotaire, O. (2019). Diet and the Role of Food in Common Gastrointestinal Diseases. Med. Clin. North Am. 103(1): 101-110.

Pearlman, M., Obert, J., and Casey, L. (2017). The Association Between Artificial Sweeteners and Obesity. Curr. Gastroenterol. Rep. 19: 64

Pepino, M.Y. (2015). Metabolic effects of non-nutritive sweeteners. Physiol. Behav. 152: 450-455.

Pereira, M.A. (2013). Diet beverages and the risk of obesity, diabetes, and cardiovascular disease: a review of the evidence. Nutr. Rev. 71(7) 433-440.

Raben, A., and Richelsen, B. (2012). Artificial sweeteners: A place in the field of functional foods? Focus on obesity and related metabolic disorders. Curr. Opin. Clin. Nutr. Metab. Care. 15(6): 597-604.

Ramos-Lopez, O., Panduro, A., Martinez-Lopez, E., and Roman, S. (2016). Sweet taste receptor TAS1R2 polymorphism (VAl191VAI) is associated with a higher carbohydrate intake and hypertriglyceridemia among the population of west Mexico. Nutrients. 8(2): 101.

Running, C.A. (2018). Oral sensations and secretions. Physiol. Behav. 193: 234-237.

Sakurai, K., Lee, E.Y., Morita, A., Kimura, S., Kawamura, H., Kasamatsu, A., Shiiba, M., Yabe, D., Yokote, K., and Miki, T. (2012). Glucagon-like peptide- 1 secretion by direct stimulation of $L$ cells with luminal sugar vs non-nutritive sweetener. J. Diabetes Investig. 3(2): 156-163.

Sakurai, M., Nakamura, K., Miura, K., Takamura, T., Yoshita, K., Nagasawa, S.Y., Morikawa, Y., Ishizaki, M., Kido, T., Naruse, Y., Suwazono, Y., Sasaki, S., and Nakagawa, H. (2014). Sugar-sweetened beverage and diet soda consumption and the 7-year risk for type 2 diabetes mellitus in middle-aged Japanese men. Eur. J. Nutr. 53(1): 251-258.

Sanematsu, K., Kitagawa, M., Yoshida, R., Nirasawa, S., Shigemura, N., and Ninomiya, Y. (2016). Intracellular acidification is required for full activation of the sweet taste receptor by miraculin. Sci. Rep. 6: 22807.

O'Donnell, K., and Kearsley, M. (2012). Sweeteners and Sugar Alternatives in Food Technology (2th ed). John Wiley and Sons, pp. 383-396.

Servant, G., Tachdjian, C., Li, X., and Karanewsky, D.S. (2011). The sweet taste of true synergy: Positive allosteric modulation of the human sweet taste receptor. Trends Pharmacol. Sci. 32(11): 631-636.

Servant, G., Tachdjian, C., Tang, X.-Q., Werner, S., Zhang, F., Li, X., Kamdar, P., Petrovic, G., Ditschun, T., Java, A., Brust, P., Brune, N., DuBois, G.E., Zoller, M., and Karanewsky, D.S. (2010). Positive allosteric modulators of the human sweet taste receptor enhance sweet taste. Proc. Natl. Acad. Sci. 107(10): 4746-4751.

Shankar, P., Ahuja, S., and Sriram, K. (2013). Non-nutritive sweeteners: Review and update. Nutrition 29(11-12): 1293-1299.

Shintani, T., Yamada, T., Hayashi, N., lida, T., Nagata, Y., Ozaki, N., and Toyoda, Y. (2017). Rare Sugar Syrup Containing d -Allulose but Not HighFructose Corn Syrup Maintains Glucose Tolerance and Insulin Sensitivity Partly via Hepatic Glucokinase Translocation in Wistar Rats. J. Agric. Food Chem. 65(13): 2888-2894.

Shirazi-Beechey, S.P., Daly, K., Al-Rammahi, M., Moran, A.W., and Bravo, D. (2014). Role of nutrient-sensing taste 1 receptor (T1R) family members in gastrointestinal chemosensing. Br. J. Nutr.111(S1): S8-S15.

Smith, K.R., Hussain, T., Karimian Azari, E., Steiner, J.L., Ayala, J.E., Pratley, R.E., and Kyriazis, G.A. (2016). Disruption of the sugar sensing receptor T1R2 attenuates metabolic derangements associated with dietinduced obesity. Am. J. Physiol. - Endocrinol. Metab. 310: E688-698.

Soldo, T., Blank, I., and Hofmann, T. (2003). (+)-(S)-alapyridaine - A general taste enhancer? Chem. Senses. 28(5): 371-379.

Stanhope, K.L. (2016). Sugar consumption, metabolic disease and obesity: The state of the controversy. Crit. Rev. Clin. Lab. Sci. 53(1): 52-67.

Stearns, A.T., Balakrishnan, A., Rhoads, D.B., and Tavakkolizadeh, A. (2010). Rapid upregulation of sodium-glucose transporter SGLT1 in response to intestinal sweet taste stimulation. Ann. Surg. 251(5): 865-871.

Steinert, R.E., Frey, F., Töpfer, A., Drewe, J., and Beglinger, C. (2011). Effects of carbohydrate sugars and artificial sweeteners on appetite and the secretion of gastrointestinal satiety peptides. Br. J. Nutr. 105(09): 1320-1328.

Steinert, R.E., Gerspach, A.C., Gutmann, H., Asarian, L., Drewe, J., and Beglinger, C. (2011). The functional involvement of gut-expressed sweet taste receptors in glucose-stimulated secretion of glucagon-like peptide-1 (GLP-1) and peptide YY (PYY). Clin. Nutr. 30(4): 524-532.

Suez, J., Korem, T., Zeevi, D., Zilberman-Schapira, G., Thaiss, C.A., Maza, O., Israeli, D., Zmora, N., Gilad, S., Weinberger, A., Kuperman, Y., Harmelin, A., Kolodkin-Gal, I., Shapiro, H., Halpern, Z., Segal, E., and Elinav E. (2014). Artificial sweeteners induce glucose intolerance by altering the gut microbiota. Nature 514(7521): 181-186.

Sylvetsky, A.C., Brown, R.J., Blau, J.E., Walter, M., and Rother, K.I. (2016). Hormonal responses to non-nutritive sweeteners in water and diet soda. Nutr. Metab. (Lond). 13(1): 71.

Tachdjian, C., Karanewsky, D.S., Tang, X.Q., Chen, Q., Leeming, P., Rashid, T., Levin, D., and Senomyx Inc. (2013). Processes and intermediates for making sweet taste enhancers. U.S. Patent 8,586,733.

Temussi, P.A. (2002). Why are sweet proteins sweet? Interaction of brazzein, monellin and thaumatin with the T1R2-T1R3 receptor. FEBS Letters 526(1-3): 1-4.

Tu, D., Luo, Z., Wu, B., Ma, X., Shi, H., Mo, C., Huang, J., and Xie, W. (2017). Developmental, chemical and transcriptional characteristics of artificially pollinated and hormone-induced parthenocarpic fruits of Siraitia grosvenorii. RSC Advances. 7(20): 12419-12428.

U.S. Services, U.S. Department of Health and Human, U.S. Department of Agriculture. (2015). 2015-2020 Dietary Guidelines for Americans. 8th edition.

U.S. Food and Drug Administration. (2018). Food Additives and Ingredients: Additional Information about High-Intensity Sweeteners Permitted for Use in Food in the United States. https://www.fda. gov/food/ingredientspackaginglabeling/foodadditivesingredients/ ucm397725.htm.

Valentin, D., Chrea, C.a.n.d.N.g.u.y.e.n., and D, H. (2006). Taste-odour interactions in sweet taste perception. Optimising Sweet Tast. Foods 66-84.

Winnig, M., Bufe, B., Kratochwil, N.A., Slack, J.P., and Meyerhof, W. (2007). The binding site for neohesperidin dihydrochalcone at the human sweet taste receptor. BMC Struct. Biol. 7: 1-12.

Wölnerhanssen, B.K., Cajacob, L., Keller, N., Doody, A., Rehfeld, J.F., Drewe, J., Peterli, R., Beglinger, C., and Meyer-Gerspach, A.C. (2016). Gut hormone secretion, gastric emptying, and glycemic responses to erythritol and xylitol in lean and obese subjects. Am. J. Physiol.-Endocrinol. Metab. 310(11): E1053-E1061.

Xu, H., Staszewski, L., Tang, H., Adler, E., Zoller, M., and Li, X. (2004). Different functional roles of T1R subunits in the heteromeric taste receptors. Proc. Natl. Acad. Sci. 101(39): 14258-14263.

Yang, Q., Zhang, Z., Gregg, E.W., Flanders, W.D., Merritt, R., and Hu, F.B. (2014). Added sugar intake and cardiovascular diseases mortality among us adults. J JAMA Intern. Med. 174(4): 516-524.

Yoshida, R., Niki, M., Jyotaki, M., Sanematsu, K., Shigemura, N., and Ninomiya, Y. (2013). Modulation of sweet responses of taste receptor cells. Semin. Cell Dev. Biol. 24(3): 226-231.

Yoshida, R., Ohkuri, T., Jyotaki, M., Yasuo, T., Horio, N., Yasumatsu, K., Sanematsu, K., Shigemura, N., Yamamoto, T., Margolskee, R.F., and Ninomiya, Y. (2010). Endocannabinoids selectively enhance sweet taste. Proc. Natl. Acad. Sci. 107(2): 935-939.

Young, R.L., Chia, B., Isaacs, N.J., Ma, J., Khoo, J., Wu, T., Horowitz, M., and Rayner, C.K. (2013). Disordered control of intestinal sweet taste receptor expression and glucose absorption in type 2 diabetes. Diabetes. 62(10): 3532-3541.

Young, R.L., Sutherland, K., Pezos, N., Brierley, S.M., Horowitz, M., Rayner, C.K., and Blackshaw, L.A. (2009). Expression of taste molecules in the upper gastrointestinal tract in humans with and without type 2 diabetes. Gut. 58(3): 337-346. 